\title{
The United States is not the world: Response to Zeller et al's call for discussion on nicotine regulation
}

Robert West, Department of Behavioural Science and Health, University College London, Gower Street, London WC1E 6BT robertwest100@gmail.com

The US Food and Drug Administration has set itself a major challenge: to drastically reduce the harms from tobacco use in the US by making smoked tobacco products less addictive and promoting addictive nicotine delivery systems that carry substantially lower risk than smoked tobacco (1).

Here I just want to draw attention to the importance of avoiding US-centrism. In the context of a set of proposals from the US Food and Drug Administration this is more understandable than in most cases where it occurs but it is still a problem. Zeller begins by stating 'Nicotine does not directly cause the estimated 480,000 deaths each year from smoking-related diseases'. This sentence should have ended 'in the US'. On its own one might let this go but in the field of tobacco control and the science and epidemiology of tobacco use, this oversight is symptomatic of a mind-set that is highly prevalent and is compounded by a tendency to disregard research from overseas. As a journal editor, I constantly have to add 'in the US' to statements that are patently false for $90 \%$ of the world's population; and I regularly have to remind authors of similar previous research conducted outside the US.

How much does this matter? Here are just two examples of why it matters relating to the proposals under consideration.

First, the document focuses on cigarettes, but cigarettes are only one form of tobacco use that dominate the US tobacco market. In India, we see a high prevalence of many other forms of tobacco use. These include 'bidis' (very cheap smoked tobacco products manufactured in small, largely unregulated facilities that can be expected to be at least as harmful as cigarettes) and smokeless tobacco products that vary hugely in toxicity (2). For its part, the US has seen a substantial increase in use of cigarillos and small cigars that we could expect to be as harmful as cigarettes but which are ignored in the headline smoking prevalence figures creating a false impression of the progress that is being made in tobacco control (3). Other countries that may be considering adopting the FDA approach will need to consider the profile of different forms of tobacco use in their jurisdiction and their relative toxicities. When they do, they might come to very different conclusions about the merits and demerits of the FDA approach. The US regulators may wish to take a closer look at the variety of different forms of tobacco used in other countries, including low nitrosamine snus in Sweden, in the mix of products it wishes to promote.

Secondly, the US is unusual among advanced industrialised countries with regard to the hegemony of commercial vested interests in formation of policies and regulation. All capitalist countries experience this to some degree but in the US it is elevated almost to a religion. This is most starkly evident in the constitutional freedom of companies to promote products in ways that to an overseas visitor look highly manipulative. The same is true with regard to candidates for election and even policies such as are being proposed here. It is shocking for Europeans to see the kind of deceptive advertising that is permitted in the US. Appreciating this from a non-US perspective could help in deciding whether, or how far, policies that are well-intended can be subverted or derailed by commercial vested interests. For example, when trying to get the concentrations of nicotine below 'addictive' thresholds in tobacco products it seems to this outsider that achieving such a goal in the current commercial and political culture in the US is highly improbable. Much more probable is that the attempt will get transformed or abandoned before the threshold is reached, during which time a lot of smokers will have been exposed to higher levels of toxins from their tobacco products as they strive the maintain their customary nicotine intake from products with reduced nicotine yields. 
So my main suggestion as this enterprise evolves is to put in place mechanisms for overcoming the tendency for US-centric thinking and to place a large health warning on the proposals that these may well not be relevant in other jurisdictions.

\section{References}

1. Zeller, M. (2018) The future of nicotine regulation: key questions and challenges. Nicotine \& Tobacco Research. In Press.

2. Sarkar B, Reddy S (2012) Priorities for tobacco control research in India. Addiction, 107, 2066-8.

3. Hitchman, S, Pearson J, Villanti, A. (2017) The need for more nuance in headline adult cigarette smoking prevalence estimates. Addiction, 112, 1327-1328. 